\title{
Editorial
}

\section{¿Por qué hacer ciencia (básica) en Colombia?: la visión muy personal de un "científico" colombiano}

\author{
BORIS A. RODRÍGUEZ R. \\ Instituto de Física, Facultad de Ciencias Exactas y Naturales, \\ Universidad de Antioquia UdeA; Calle 70 No. 52-21 \\ Medellin-Colombia
}

Generalmente en el salón de clases, frente a estudiantes de primer semestre de Física o Astronomía, o estudiantes de quinto semestre de Mecánica clásica o Física matemática, o aún frente a estudiantes que están finalizado sus carreras o incluso en los seminarios "Landau o alemanes" con mis estudiantes de maestría y doctorado, intento ser optimista frente al reto que tienen de desarrollarse como científicos en un país como Colombia. Intento convencerlos desde la pasión por el conocimiento de la importancia que tiene hacer ciencia, del valor singular que como ciudadanos de estas tierras macondianas tiene el de participar de una aventura cultural global del conocimiento, intento convencerlos de que podemos alcanzar las más altas cumbres sin olvidar nuestro contexto y nuestra historia. Tampoco pretendo actuar como un romántico de la ciencia y la naturaleza como nuestros héroes del siglo XIX, y también les hablo de sus peligros y de sus abismos. De cómo a veces los hombres y mujeres supuestamente más inteligentes, pueden dejarse manipular por los políticos, la sociedad y sus poderes, y por ejemplo participar en la construcción colectiva de la Bomba atómica, o de cómo el sistema social más eficiente y "deshumanizado" creado por el hombre, el capitalismo, se alimenta y se desarrolla gracias a los avances técnicos posibilitados por la ciencia. En medio de esta posición optimista con los estudiantes, crecen cada vez más las sombras y las preguntas sobre el futuro de la actividad científica en Colombia. Veamos algunos de estos nubarrones, preguntas o inquietudes.

Recientemente, con grandes anuncios y despliegue de publicidad, el gobierno declaró que una de las "locomotoras" para el desa- 
rrollo de Colombia era la Ciencia la Tecnología y la Innovación, y para ello promulgó las leyes 1286 del 23 de enero de 2009, de Ciencia, Tecnología e Innovación (CT\&I) y que transformó a COLCIENCIAS en Departamento Administrativo; y la 1530 del 17 de mayo 2012, por la cual se regula la organización y seguimiento del Sistema General de Regalías. La situación de optimismo entre la comunidad científica fue creciente porque pensamos que por fin íbamos a tener una política de estado en CT\&I y que esa política iba a contar con recursos. Sin embargo, el desarrollo de las leyes y la situación actual, muestra que este optimismo era desbordado y que debemos continuar con el espíritu crítico y alerta sobre el desarrollo de las políticas de CT\&I. Algunas de las críticas más agudas, es que Colombia como estado y nación sigue sin contar con una política nacional de ciencia y tecnología ${ }^{1}$. Es decir, Colombia como estado no ha tomado la decisión de qué aspectos jalonar o priorizar para su desarrollo a mediano o largo plazo, y el asunto no es que los científicos colombianos no sepamos en qué dirección avanzar $^{2}$, sino que en las instancias de decisión política y en la misma sociedad, el discurso de la ciencia y el conocimiento no es sino un remedo o una caricatura que usan a su acomodo.

Por ejemplo, las altas instancias del estado usan el lenguaje de la ciencia para quedar bien con los organismos y fondos internacionales, y el hombre de la calle usa la ciencia en la forma de dispositivos tecnológicos que le faciliten la vida sin siquiera preguntarse cuáles son las condiciones de posibilidad para que un artefacto de estos exista. Estamos frente a una situación no muy diferente a la Colombia de la Colonia, en donde los avances de la $\mathrm{Me}$ -

$1 \mathrm{Al}$ respecto pueden verse los artículos: Beatriz Suárez de Sarmiento, "Financiación y Regionalización de la Investigación en Colombia: ¿Qué podemos esperar?" Medicina Vol. 34 pp. 370-386, diciembre de 2012. Jorge Mahecha "Investigación sin ciencia básica: una colombianada más", Periódico Alma Mater No. 612, Agosto 2012, p. 17

2 Sin entrar en detalles podríamos decir que Colombia debe y puede realizar Investigación Básica, Aplicada y desarrollos tecnológicos en: Productos naturales desde industria farmacéutica hasta biotecnología, energía solar, eólica y otras energías limpias, recursos hídricos y aprovechamiento del agua, medio ambiente y biodiversidad, clima y oceanografía. Es interesante notar como Colombia sigue graduando miles de abogados, administradores de empresas, etc., y que las profesiones con menor demanda son las ciencias agrarias y las ciencias básicas. 
cánica newtoniana fueron traídos por José Celestino Mutis 100 años después, y por lo tanto, podemos afirmar con el profesor Jaime Rodríguez Lara que Colombia no es solo una nación que pasó la mitad del siglo XX de espaldas a los extraordinarios avances de la ciencia (la física, la química, la biología, etc.) sino que prácticamente en pleno siglo XXI Colombia sigue siendo una nación profundamente acientífica, con un analfabetismo científico alarmante y con una sociedad y unas élites políticas que no valoran la ciencia en el desarrollo de la vida individual y de una sociedad ${ }^{3}$. Retomando las palabras del profesor Moisés Wasserman:

\begin{abstract}
No es extraño ver sobre el escritorio de importantes hombres de gobierno (en informes televisados a toda la nación), una pirámide de cuarzo que 'concentra las buenas energías cósmicas' en su dueño y le mejora las posibilidades de éxito en su gestión. En alguna reciente reunión en el exterior, realizada entre representantes del gobierno colombiano y un grupo guerrillero, estalló un escándalo porque los guerrilleros pensaban que estaban siendo espiados con sofisticados equipos electrónicos. No los estaban espiando, les estaban midiendo el 'aura'. Un bien relacionado 'bioenergético' estaba ayudando a diseñar las estrategias de la negociación y para ello le resultaba fundamental conocer el aura de los negociadores del otro bando. Un importante político colombiano afirmaba (con titular de varias columnas en la primera página del periódico de mayor circulación): "aunque no hay pruebas, todo el mundo sabe que la Fiscalía, la Procuraduría y la Defensoría del Pueblo están infiltradas por la guerrilla". ¿Qué extraño mecanismo permite a todo el mundo saber algo sin que exista ninguna prueba? ${ }^{4}$
\end{abstract}

3 Un buen indicador de nuestra cultura científica son las secciones de "ciencias" de los medios masivos de comunicación. Cuando las hay, están dedicadas a dar noticias del tipo "Un estudio de la Universidad XXX (extranjera y muy importante) COMPROBÓ que el uso de YYY (algo desde cotidiano hasta útil) es muy perjudicial (o no) para la salud". Dicho de otra forma, los medios de comunicación confunden correlación estadística con una relación causal. En el plano social encontramos ex-ministros y ex-gerentes de empresas de energía que le hacen publicidad a la "medicina cuántica", etc. Para ejemplificar los mitos culturales de la física cuántica véase el artículo B. A. Rodríguez "Mecánica Cuántica: representaciones culturales, mitos y sus fundamentos", Mínima Acción, No. 2, pp. 21-23, 2013.

4 M. Wasserman, "Por qué investigar en Colombia" En Reflexiones sobre la ciencia y la tecnología: Colombia al iniciar el siglo XXI, Colección Memorias No. 11. Academia Colombiana de Ciencias Exactas Físicas y Naturales, pp. 99-116, 2001; M. Wasserman, "Sobre la importancia de investigar en Colombia, un país subdesarrollado", Biomédica, Vol. 21, pp. 13-24, 2001. 
Esta actitud frente a la ciencia incluso es compartida por ciertas élites culturales de la tradición "literaria" colombiana. Un ejemplo muy reciente es el debate alrededor del artículo "Ciencias Inútiles" del reconocido académico e historiador Jorge Orlando Melo en donde afirma "... Como el desarrollo científico ha sido en otras partes causa del crecimiento, el país ha estimulado la investigación científica en las universidades, pero es una ciencia que tiene poco que ver con la realidad del país..."5.

Uno de los aspectos donde la ciencia puede hacer sus mayores aportes en una sociedad como la Colombiana, es justamente en la educación. El gran fracaso de la educación en Colombia (y en otros países) es que una educación en donde supuestamente se privilegia la enseñanza de las matemáticas, las ciencias y el lenguaje ${ }^{6}$ no sea capaz de formar un ciudadano medianamente apto para decidir por sí mismo, un ciudadano autónomo y capaz de pensar por sí mismo, de tomar las riendas de su proyecto de vida y actuar en consecuencia. Y el ser crítico es el carácter más importante de una enseñanza científica. Es decir, no se puede enseñar ciencias como se enseña una religión o una ideología, porque en las ciencias no tenemos verdades inamovibles o dogmas, y una enseñanza verdaderamente científica es necesariamente crítica. El primer movimiento de la ciencia y un científico es el de dudar, el de valorar cuidadosamente los hechos y lo que se le está afirmando, y acto después, el de ponerse a pensar por sí mismo, el de tratar de comprender, de entender, de solucionar el problema.

Esta actitud crítica de la ciencia es tan fuerte, que se entiende que desde los extremos políticos del país se produzcan discursos en contra de ella. Por ejemplo, un exdirector de COLCIENCIAS que además fue exrector de una de las principales universidades públi-

5 El artículo del profesor Melo fue publicado el 13 de marzo de 2013 en las páginas editoriales de El tiempo. Puede verse reproducida en el portal http://www.universidad.edu.co en donde además está la respuesta de Hernán Jaramillo Salazar. Ver el enlace http://goo.gl/TUHWq

6 Aunque esto ni siquiera es cierto hoy en día. Basta con ver el currículo de los niños de las escuelas oficiales y en donde además de que la jornada escolar está muy reducida ven hasta 15 asignaturas: inglés, computación, ética, sociales, español, matemáticas, educación física, ciencias, religión, educación física, artísticas, etc. Es decir hoy en día ni siquiera podemos afirmar que la educación tenga un carácter mayoritariamente científico. 
cas del país, se mostró preocupado por el alarmante crecimiento de las publicaciones de artículos de científicos colombianos, según este exdirector, los profesores de las universidades colombianas que hacen investigación están más preocupados por escribir artículos científicos que en resolver los problemas del país. Por el otro extremo, del espectro ideológico, uno puede ver en las paredes de cualquier pared de Universidad Pública, que se reclama una ciencia por el pueblo y para el pueblo?

Es curioso notar como extremos ideológicos tan irreconciliables en el plano político se ponen de acuerdo para demeritar y atacar la labor de la ciencia. Además es curioso, porque la actividad científica en el país apenas si existe. Por ejemplo, el número de artículos científicos en publicaciones internacionales es tan exiguo, que Colombia no aparece en el mapa de la ciencia a nivel mundial, esto puede verse dramáticamente en la Fig. 1. A nivel latinoamericano apenas alcanzamos el $2 \%$ o $5 \%$ de la producción que consiguen países como Brasil o México. Para ahondar en la comparación, la revista más importante en la física desde la segunda mitad del siglo XX es la "Physical Review Letters" publicada por la American Physical Society. En los últimos 60 años se han publicado aproxi-

$7 \quad$ Por ejemplo en las paredes de las universidades públicas es común encontrar la llamada Declaración del 1969 de los Estudiantes de Tucumán, en la que entre otras cosas además de hacer una declaración libertaria sobre la universidad se afirma que “... No queremos cátedras de Ciencias donde los tecnócratas cientifistas mienten que los esquemas de la física, la química, la biología y las matemáticas son herramientas suficientes para construir un mundo moderno, ignorando estructuras sociales que las hacen estériles... Queremos terminar la universidad academicista de las clases magistrales, de los repetidores incansables de textos muertos, del fetiche de la elaboración científica de la metrópolis, en función de necesidades culturales y técnicas divorciadas de nuestra realidad de atraso y dependencia. Queremos una universidad científica. Pero creemos que la integración de la universidad al pensamiento científico universal no será lograda por la adopción mecánica de conocimientos acabados en una realidad distinta, sino por la investigación y solución los problemas de nuestra propia realidad". Aunque uno puede estar de acuerdo con el tono libertario de la Declaración, se sorprende por el papel que le asocian a las ciencias. ¿Acaso la ciencia no es un discurso universal? ¿Las leyes de la física, química, biología son distintas para nuestros países y por lo tanto debemos crear nuevas leyes para resolver nuestros problemas? Como lo mencionamos antes, parece que los estudiantes confunden la ciencia con el modelo social prevalente, el cual por supuesto hace un uso prioritario de las técnicas desarrolladas por la ciencia. 
madamente 10 a 20 artículos científicos en esta revista, cuyo origen es de universidades colombianas y con investigadores mayoritariamente colombianos en la lista de autores. Esta revista publica 50 números al año, cada uno con 100 o más artículos. Es decir, de unos 5000 artículos que aparecen en Physical Review Letters aproximadamente al año, uno o ninguno son colombianos. Otro aspecto que habla de nuestro avance científico es que tan solo unos 100 o 200 colombianos están capacitados para leer estos artículos científicos.

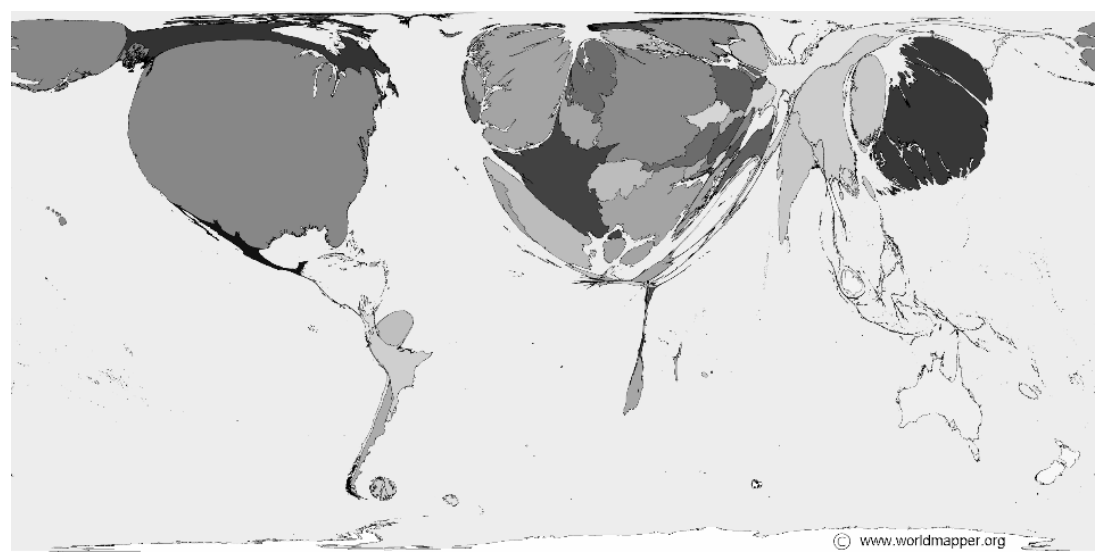

Fig. 1. Mapa de la ciencia a nivel mundial. El área de cada país se grafica proporcional al número de artículos científicos publicados en el 2001. Como podemos ver, Colombia apenas logra aparecer en el mapa, mientras Japón, Europa y los EEUU son verdaderamente "obesos". Tomado de http://www.sasi.group.shef.ac.uk/worldmapper/index.html

Reflexionando sobre estos hechos 8 , los cuales son posibles llevar a todas las áreas de la ciencia ${ }^{9}$, no se entiende una declaración

8 Esta es una característica importante de la ciencia. Intenta interpretar, modelar, entender, teorizar sobre los hechos. Pero no intenta "torcer" los hechos, negarlos, ideologizarlos. La historia de la ciencia está construida sobre estos relatos. Basta recordar quienes se negaban a mirar por el telescopio de Galileo o de los gérmenes de Pasteur, etc.

9 El ejercicio que puede hacerse con la física, prácticamente puede hacerse con cualquier área de la ciencia. La incidencia de Colombia en las revistas más importantes de cada área es muy escasa. Otro ejemplo muy notable es la casi nula participación de la ciencia colombiana en revistas como Nature o Science 
tan desafortunada como la de un exdirector de COLCIENCIAS. Es evidente que los científicos y la ciencia colombiana no publican el número suficiente de artículos, entre otras cosas porque tiene un número insuficiente de científicos y porque la inversión en CT\&I a duras penas alcanza el $0.6 \%$ del PIB ${ }^{10}$. Colombia necesita multiplicar por lo menos por $10 \mathrm{su}$ capacidad científica si quiere aspirar a ser una nación soberana, digna, con ciudadanos que decidan su futuro. Los científicos colombianos, además de nuestro esfuerzo en la educación de nuestros conciudadanos, debemos seguir incorporándonos, desde nuestra visión particular, con nuestros problemas, en el contexto de la ciencia mundial.

las más importantes en la actualidad en todas las áreas de la ciencia.

10 Antes de la Ley de Regalías, el porcentaje de inversión en CT\&I era de apenas el $0.16 \%$. Con la inyección de las regalías se estima que entran unos 800.000 millones de pesos al año a lo largo de una década. Con esto llegaríamos al $0.6 \%$ del PIB, aun por debajo de la media mundial. Además como se ha señalado en la referencia 1, no hay razones para ser optimistas: El dinero de las regalías está esencialmente controlado por las agendas políticas regionales. 